\title{
0 papel da catastrofização da dor no prognóstico e tratamento de idosos com osteoartrite de joelho: uma revisão crítica da literatura
}

\author{
The role of pain catastrophizing in prognosis and treatment of elderly with knee osteoarthritis: a \\ critical literature review
}

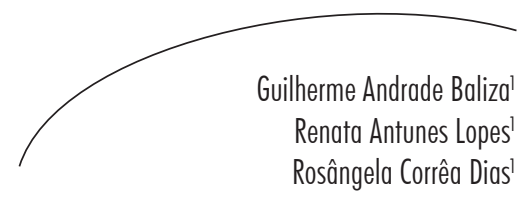

Resumo

A artroplastia total de joelho, procedimento realizado em pacientes com osteoartrite severa, pode levar à melhora na dor, na função física e na qualidade de vida, porém alguns indivíduos apresentam resultados negativos após esse procedimento. A catastrofização da dor tem sido apontada como o preditor psicológico mais importante para os piores resultados após a cirurgia. Assim, o objetivo deste estudo foi realizar uma revisão da literatura acerca da influência da catastrofização da dor no prognóstico e tratamento de indivíduos idosos com osteoartrite de joelho. Foi realizada uma busca da literatura nas bases de dados Medline via PubMed, Embase, Cochrane, Scopus, Science Direct, MedCarib, SciELO, LILACS e Google Acadêmico, a partir do primeiro registro até junho de 2012. A busca resultou num total de 2.227 artigos e, após exclusão por título, resumo e leitura integral, foram selecionados seis estudos. Todos os artigos mostraram como característica comum de tratamento a realização da artroplastia total de joelho. Apesar da análise de outras variáveis como depressão, ansiedade, gravidade da dor, incapacidade, somente a catastrofização revelou ser a variável que contribui significativamente para os resultados da recuperação após a artroplastia, contribuindo para maior tempo de permanência no hospital, maior intensidade de dor e piora na funcionalidade. É necessário que medidas básicas para a identificação dos indivíduos que catastrofizam mais sejam aplicadas e, assim, intervenções psicossociais podem ser necessárias para promover uma recuperação mais positiva em indivíduos que apresentam perfil de risco psicossocial, diminuindo o tempo de hospitalização.

\section{Abstract}

Total knee arthroplasty performed on patients with severe osteoarthritis can lead to improvement in pain, physical function and quality of life. However, some individuals have negative results after this procedure. Pain catastrophizing has been identified as the most important psychological predictor for worse outcomes after surgery. This study aimed to review the literature about the influence of pain catastrophizing on

Palavras-chave: Catastrofização. Dor. Osteoartrite do Joelho. Idoso.

Departamento de Fisioterapia, Escola de Educação Física e Terapia Ocupacional. Universidade Federal de Minas Gerais. Belo Horizonte, MG, Brasil. 
prognosis and treatment of elderly subjects with knee osteoarthritis. Literature search was performed in Medline via PubMed, Embase, Cochrane, Scopus, Science Direct, MedCarib, SciELO, LILACS and Google until June 2012. The search yielded a total of 2,227 articles, and after exclusion by title, abstract and full text, six studies were included. All items shown as a common feature of treatment as TKA surgery. Although the analysis of other variables such as depression, anxiety, pain severity and disability, catastrophizing was considered the only variable that significantly contributes to the results of the recovery after arthroplasty, contributing to longer hospital stays, more pain and deterioration in functionality. It is necessary to put basic measures into practice to identify individuals who are more catastrophizers and thus psychosocial interventions may be needed to promote a more positive recovery in individuals presenting a profile of psychosocial risk, reducing the length of hospitalization.
Key words:

Catastrophization. Pain. Osteoarthritis, Knee. Elderly.

\section{INTRODUÇÃ̃o}

Mantidas as tendências dos parâmetros demográficos para a população do Brasil, a sociedade percorrerá velozmente um caminho rumo a um perfil demográfico cada vez mais envelhecido. ${ }^{1}$ Com o envelhecimento populacional, ocorrerá aumento da prevalência das doenças crônico-degenerativas, dentre elas a osteoartrite $(\mathrm{OA}))^{2,3}$ Apesar de não existirem dados precisos sobre sua prevalência, acredita-se que a $\mathrm{OA}$ atinja cerca de 15 milhões de pessoas no país. Nos membros inferiores, a OA avançada é frequentemente caracterizada por dor intensa, rigidez, edema e restrições de mobilidade, sendo a segunda maior causa de faltas ao trabalho e aposentadoria por invalidez. ${ }^{2-5}$

Segundo o Colégio Americano de Reumatologia, ${ }^{6}$ há três formas de tratamento principais para a OA: o tratamento não farmacológico, que consiste na utilização de programas educativos; prescrição individualizada, pelo fisioterapeuta, de exercícios terapêuticos e prescrição do uso de órteses; tratamento farmacológico para diminuição de dor e processo inflamatório; e tratamento cirúrgico para os pacientes que apresentam grau mais severo de OA com comprometimento progressivo da independência das atividades de vida diária e/ou falha no tratamento conservador. ${ }^{2,3,7}$
O tratamento cirúrgico mais comum é a artroplastia total de joelho (ATJ), procedimento mais efetivo para os pacientes com OA de joelho severa e que não responderam ao tratamento conservador. ${ }^{3,5}$ No entanto, há um número considerável de indivíduos que apresentam pequena melhora da dor e, em alguns casos, piora da dor e funcionalidade após a ATJ. ${ }^{5,8-10}$ Brander et al. ${ }^{11}$ mostraram que $19 \%$ dos 116 pacientes estudados relataram dor moderada a grave seis meses após a cirurgia, assim como em Hawker et al. ${ }^{12}$ Por esses motivos, muitas pesquisas estão dando destaque à análise das causas da dor persistente e da limitação de funcionalidade dos pacientes submetidos à ATJ., 2,5,13-15

Os pontos mais discutidos e consistentes são os preditores psicossociais, dentre os quais se destaca a catastrofização. ${ }^{16-25}$ A catastrofização da dor é definida como orientação negativa a determinados estímulos dolorosos e tem sido relacionada a desfechos funcionais negativos e dor. ${ }^{26}$ Embora os mecanismos específicos pelos quais a catastrofização pode influenciar a dor ainda não tenham sido determinados, parece que esse construto apresenta influência direta sobre os mecanismos neurofisiológicos envolvidos no processamento da dor. ${ }^{27}$ A catastrofização da dor é caracterizada por três componentes: a ruminação, caracterizada como uma tendência a se concentrar excessivamente nas sensações de 
dor por pensamentos repetitivos; a magnificação, que consiste na ampliação do desprazer; e o desamparo, quando o indivíduo percebe-se como sendo incapaz de controlar os sintomas de dor. ${ }^{26-29}$

Apesar de alguns autores ressaltarem a influência da catastrofização na dor e na funcionalidade, Kovacs et al. ${ }^{30}$ demonstraram que a influência das crenças e da catastrofização da dor foi pequena na amostra estudada, evidenciando resultados conflitantes.

Assim, dado o aumento da prevalência de doenças crônico-degenerativas decorrentes do envelhecimento populacional, maior utilização de procedimentos cirúrgicos como a ATJ para tratamento de $\mathrm{OA}$ e relatos de desfechos funcionais e clínicos negativos após esses procedimentos, faz-se necessário investigar possíveis fatores que possam influenciar no prognóstico e tratamento dos pacientes após ATJ. ${ }^{31,32}$ Dessa forma, o objetivo deste estudo foi realizar uma revisão da literatura acerca da influência da catastrofização da dor no prognóstico e tratamento de indivíduos idosos com osteoartrite (OA) de joelho.

\section{METODOLOGIA}

Foi realizada busca na literatura nas bases de dados Medline via PubMed, Embase, Cochrane, Scopus, Science Direct, MedCarib, SciELO, LILACS e Google Acadêmico, a partir do primeiro registro até junho de 2012. Os descritores utilizados na busca nas bases de dados foram selecionados a partir do Medical Subject Headings (MeSH) e foram os seguintes: catastrophizing ou catastrophization, knee osteoarthritis, elderly e seus correlatos em português. Não houve restrição quanto à data de publicação dos artigos. Também foram utilizados artigos do banco de dados pessoal dos autores e referências citadas pelos artigos encontrados nas bases de dados.
Para serem incluídos, os estudos deveriam investigar indivíduos idosos com osteoartrite de joelho que catastrofizavam a sua dor, sem restrição quanto ao procedimento cirúrgico realizado previamente ou o tempo de análise da dor após a artroplastia de joelho, nos casos de indivíduos que realizaram esse procedimento. Todos os estudos deveriam ter como um dos desfechos a catastrofização da dor, não importando a duração ou a intensidade deste sintoma. Não houve restrição quanto ao método de análise da dor ou do diagnóstico da osteoartrite. Apenas artigos publicados em inglês e português foram selecionados. Os estudos potencialmente elegíveis foram identificados por meio do título, resumo, leitura completa do artigo, do banco de dados pessoal do autor ou por meio das referências dos artigos encontrados nas bases de dados.

A busca resultou num total de 2.227 artigos, mas 2.191 foram excluídos pela leitura do título ou resumo (figura 1). Os títulos excluídos $(\mathrm{n}=2.047)$ não contemplavam a osteoartrite de joelho, mas em outras articulações como quadril, coluna e mãos. Dos resumos excluídos, 143 eram repetidos e um foi escrito em japonês. Restaram 36 artigos potencialmente elegíveis. Após leitura mais minuciosa dos resumos selecionados, 22 foram excluídos por incluírem somente grupos amostrais de adultos jovens e de meia-idade.

Assim, 14 resumos foram selecionados para checagem dos textos completos. Onze estudos foram excluídos por não investigarem a catastrofização da dor no prognóstico e/ou tratamento de indivíduos com osteoartrite de joelho, apesar de contemplarem outros fatores psicossociais (depressão, autoestima, ansiedade). Dois artigos foram incluídos a partir de busca nas referências dos artigos previamente selecionados e um estudo foi incluído a partir do banco de dados pessoal do autor. Dessa forma, foram incluídos seis artigos nesta revisão (figura 1). 


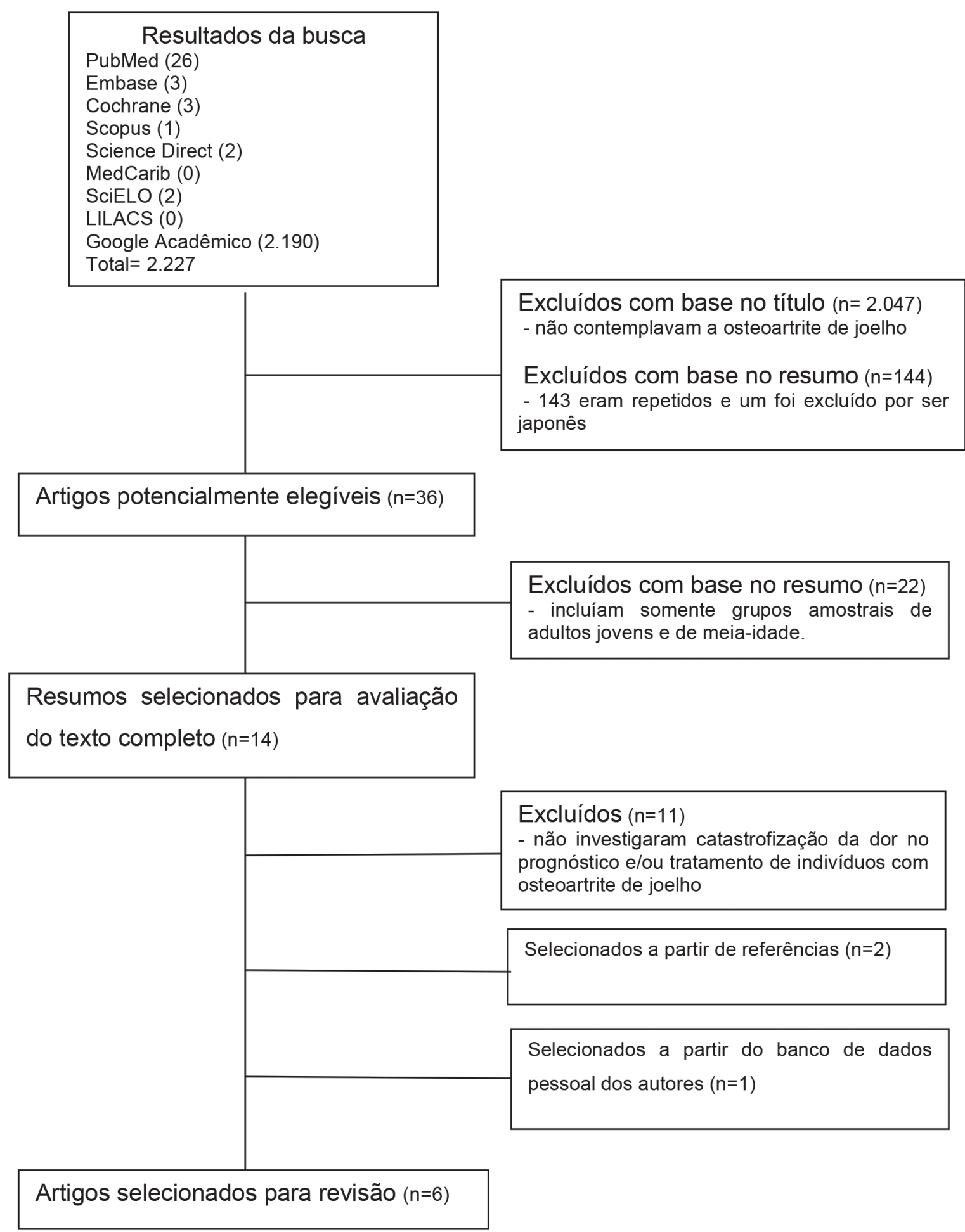

Figura 1. Progresso para seleção de estudos para revisão. 


\section{RESULTADOS}

A caracterização e análise crítica dos estudos foram realizadas a partir da extração dos seguintes dados: tipo do estudo, tamanho da amostra, média de idade da população estudada, porcentagem do gênero dominante, método de avaliação da catastrofização da dor, outras variáveis analisadas que não a catastrofização da dor, período de avaliação e acompanhamento, critérios de inclusão e exclusão de cada estudo e resultados obtidos em relação à influência da catastrofização na osteoartrite de joelho (quadros 1 e 2).

Todos os seis estudos analisados apresentaram como característica comum a realização da
ATJ como procedimento cirúrgico e eram longitudinais prospectivos, delineamento adequado para avaliação de mudanças nas variáveis ao longo do tempo. O tamanho amostral variou de 43 a 157 indivíduos e a média de idade ficou entre 61 e 71,7 anos. O sexo feminino predominou em todos os estudos, oscilando entre 58 e $70,7 \%$ da amostra total.

Nos seis estudos, outras variáveis foram analisadas além da catastrofização da dor (depressão, cinesiofobia, comorbidades, expectativas e ansiedade). O período de acompanhamento foi heterogêneo, variando de follow up curto (14 dias) a longo prazo (24 meses).

Quadro 1. Tipos de estudos, características das amostras e instrumentos de avaliação. Belo HorizonteMG, 2012.

\begin{tabular}{cccccc}
\hline \multirow{2}{*}{ Artigo } & Tipo de & \multicolumn{4}{c}{ Características da amostra } \\
\cline { 3 - 6 } & estudo & $\begin{array}{c}\text { Tamanho } \\
\text { amostral }\end{array}$ & $\begin{array}{c}\text { Média idade } \\
\text { (anos) }\end{array}$ & $\begin{array}{c}\text { Porcentagem } \\
\text { do gênero } \\
\text { dominante }\end{array}$ & $\begin{array}{c}\text { Método de } \\
\text { avaliação da } \\
\text { catastrofização }\end{array}$ \\
\hline Riddle et al. ${ }^{24}$ & $\begin{array}{c}\text { Longitudinal } \\
\text { prospectivo }\end{array}$ & 157 pacientes & 63,7 anos & $70,7 \%$ mulheres & PCS \\
\hline Sullivan et al. ${ }^{44}$ & $\begin{array}{c}\text { Longitudinal } \\
\text { prospectivo }\end{array}$ & 120 pacientes & 67 anos & $60,8 \%$ mulheres & PCS \\
\hline Witvrouw et al. ${ }^{34}$ & $\begin{array}{c}\text { Longitudinal } \\
\text { prospectivo }\end{array}$ & 43 pacientes & 61 anos $(41-79)$ & $60,5 \%$ mulheres & PCS \\
\hline Wade et al. ${ }^{35}$ & $\begin{array}{c}\text { Longitudinal } \\
\text { prospectivo }\end{array}$ & 150 pacientes & $63,5 \pm 9,9$ anos & $70,1 \%$ mulheres & PCS \\
\hline Edwards et al. ${ }^{21}$ & $\begin{array}{c}\text { Longitudinal } \\
\text { prospectivo }\end{array}$ & 43 pacientes & $71,7 \pm 7$ anos & $58 \%$ mulheres & $\begin{array}{c}\text { CSQ } \\
\text { catastrophizing }\end{array}$ \\
\hline Forsythe et al. ${ }^{22}$ & $\begin{array}{c}\text { Longitudinal } \\
\text { prospectivo }\end{array}$ & 55 pacientes & 69 anos (49- 85) & $64 \%$ mulheres & PCS \\
\hline
\end{tabular}

PCS= Pain Catastropbizing Scale (Escala de Catastrofização da Dor); CSQ catastrophizing= Coping Strategies Questionnaire Catastrophizing (Questionário de Estratégias para Enfrentamento da Catastrofização). 
Quadro 2. Variáveis analisadas, período de avaliação e critérios de seleção das amostras. Belo HorizonteMG, 2012.

\begin{tabular}{|c|c|c|c|}
\hline Artigo & $\begin{array}{l}\text { Outras variáveis } \\
\text { analisadas }\end{array}$ & $\begin{array}{l}\text { Período da } \\
\text { avaliação }\end{array}$ & Critérios de inclusão e exclusão \\
\hline Riddle et al. ${ }^{24}$ & $\begin{array}{l}\text { WOMAC (dor e } \\
\text { função); PHQ-8 } \\
\text { (depressão); Tampa } \\
\text { Scale of Kinesiophobia; } \\
\text { Comorbidades; SF-36. }\end{array}$ & $\begin{array}{l}\text { Pré-cirúrgico; } \\
6 \text { meses pós- } \\
\text { cirúrgico. }\end{array}$ & $\begin{array}{l}\text { Exclusão: operação prévia de joelho; não } \\
\text { consentimento para participação do estudo. }\end{array}$ \\
\hline Sullivan et al. ${ }^{44}$ & $\begin{array}{l}\text { WOMAC (dor e } \\
\text { função); Comorbidades; } \\
\text { Expectativas; PHQ-9 } \\
\text { (depressão); Tampa Scale } \\
\text { of Kinesiophobia. }\end{array}$ & $\begin{array}{l}\text { Pré-cirúrgico; } \\
12 \text { meses pós- } \\
\text { cirúrgico. }\end{array}$ & $\begin{array}{l}\text { Inclusão: diagnóstico primário de OA de joelho; } \\
\text { idade entre } 50 \text { e } 85 \text { anos; ser selecionado para } \\
\text { ATJ em um dos três hospitais colaboradores } \\
\text { do Canadá. Exclusão: diagnóstico de artrite } \\
\text { reumatoide; artroplastia prévia de joelho; } \\
\text { patelectomia prévia; IMC> } 45 \text { pré-operação; } \\
\text { evidências de abuso de substâncias; perda óssea } \\
\text { grande que necessite de enxerto ósseo estrutural; } \\
\text { funcionalmente limitado por doença vascular } \\
\text { periférica; fratura prévia de patela, côndilo- } \\
\text { femoral, ou platô-tibial; requerimento de ATJ } \\
\text { bilateral dentro de um ano do procedimento. }\end{array}$ \\
\hline $\begin{array}{l}\text { Witvrouw et } \\
\text { al. }^{34}\end{array}$ & $\begin{array}{l}\text { WOMAC (dor, } \\
\text { incapacidade e rigidez). }\end{array}$ & $\begin{array}{l}\text { Pré-cirúrgico; } \\
\text { 6-14 dias pós- } \\
\text { cirúrgico. }\end{array}$ & $\begin{array}{l}\text { Inclusão: selecionados para ATJ primária no } \\
\text { Hospital Universitário de Ghent entre maio de } \\
2006 \text { e janeiro de } 2007 \text {; estar disposto a participar. } \\
\text { Exclusão: operação prévia de joelho; infecções; } \\
\text { traumas; artrite reumatoide; ter mais de } 80 \text { anos; } \\
\text { ser incapaz de responder os questionários ou } \\
\text { respondê-los incompletamente. }\end{array}$ \\
\hline Wade et al..$^{35}$ & $\begin{array}{l}\text { EVA (dor); PHQ-8 } \\
\text { (depressão); PRIME- } \\
\text { MD (ansiedade); } \\
\text { Comorbidades. }\end{array}$ & $\begin{array}{l}\text { Pré-cirúrgico; } \\
\text { 2, } 6 \text { meses pós- } \\
\text { cirúrgico. }\end{array}$ & $\begin{array}{l}\text { Inclusão: falar e entender inglês; selecionado para } \\
\text { ATJ primária; consentimento para participação } \\
\text { do estudo. Exclusão: não consentimento para } \\
\text { participação do estudo; cirurgia prévia de joelho. }\end{array}$ \\
\hline Edwards et al. ${ }^{21}$ & $\begin{array}{l}\text { Global Daily Pain; } \\
\text { Nighttime Pain (gravidade } \\
\text { da dor); CES-D } \\
\text { (depressão). }\end{array}$ & $\begin{array}{l}\text { Pré-cirúrgico; } \\
1,3,6,12 \\
\text { meses pós- } \\
\text { cirúrgico. }\end{array}$ & $\begin{array}{l}\text { Inclusão: participantes com dor unilateral } \\
\text { intratável no joelho que estavam programados } \\
\text { para ATJ no Departamento de Cirurgia } \\
\text { Ortopédica de Hopkins. }\end{array}$ \\
\hline Forsythe et al. ${ }^{22}$ & $\begin{array}{l}\text { McGill Pain } \\
\text { Questionnaire (dor); } \\
\text { Queen Elizabeth II } \\
\text { Health Sciences Centre } \\
\text { (comorbidades). }\end{array}$ & $\begin{array}{l}\text { Pré-cirúrgico; } \\
\text { 3, 12, } 24 \text { meses } \\
\text { pós-cirúrgico. }\end{array}$ & Não relatados. \\
\hline
\end{tabular}

WOMAC = Western Ontario and McMaster Universities Artbritis Index; PHQ-8 = Eight-item Patient Health Questionnaire Depression Scale; SF-36 = The 36-Item Short-Form Health Survey; PHQ-9 = Nine-item Patient Health Questionnaire Depression Scale; OA = osteoartrite; ATJ = artroplastia total de joelho; IMC = índice de massa corporal; EVA = escala visual analógica de dor; PRIME-MD = Primary Care Evaluation of Mental Disorders; CES-D = Center for Epidemiologic Studies Depression Scale. 


\section{DISCUSSÃO}

Todos os seis artigos mostraram que, apesar da análise de outras variáveis como depressão, ansiedade, gravidade da dor, incapacidade, rigidez, expectativas e comorbidades, somente a catastrofização da dor revelou ser a variável que contribui significativamente para os resultados da recuperação após a ATJ. Ou seja, piores escores na PCS (Escala de Catastrofização da Dor) estão relacionados a maior tempo de permanência no hospital, maior intensidade de dor e piora na funcionalidade após a ATJ.

Segundo Sullivan et al., ${ }^{23}$ o indivíduo que catastrofiza sua dor tende a ter mais cinesiofobia (medo do movimento), mais medo de se lesionar, levando a um comportamento de desuso, incapacidade, depressão e baixa qualidade de vida. Uma vez estando deprimido e incapacitado, as chances de aumentar a catastrofização são maiores, podendo gerar um ciclo vicioso. ${ }^{21,23,33}$

Os artigos de Edwards et al. ${ }^{21}$ e Forsythe et al. ${ }^{22}$ relatam que os escores de dor diminuíram após a ATJ, mas o escore de catastrofização da dor não apresentou diminuição significativa, mostrando que essa variável permanece estável após a cirurgia, a menos que uma intervenção psicológica específica seja realizada.

$\mathrm{O}$ artigo de Witvrouw et al..$^{34}$ demonstrou que a idade e a catastrofização da dor são preditores significativos de longas permanências em hospitais após ATJ, ou seja, indivíduos mais idosos e com altos escores na escala de catastrofização da dor no período pré-operatório apresentam maiores chances de longas permanências nos hospitais pós ATJ e, por consequência, maior risco de complicações respiratórias, maior prejuízo funcional e maior risco de mortalidade.

Já os outros três artigos relatam diminuição considerável dos escores de dor e catastrofização da dor, e mostram correlações positivas entre esses construtos. De acordo com os autores dos estudos revisados, a catastrofização está fortemente relacionada com a intensidade da dor. Wade et al. ${ }^{35}$ relatam que os indivíduos são mais propensos a catastrofizarem quando a dor é mais intensa, e que tanto os fatores genéticos quanto a história autobiográfica de um indivíduo influenciam diretamente no grau de ampliação da catastrofização da dor. ${ }^{23,35}$

A artroplastia total de joelho (ATJ), cirurgia para melhorar a mobilidade articular, foi possivelmente o tipo de intervenção principal mais utilizado nos artigos revisados, devido às características da população em estudo. ${ }^{7,834} \mathrm{Em}$ sua maioria, tratava-se de idosos com intensidade de dor alta, que possivelmente possuíam comorbidades limitantes da mobilidade articular, baixa qualidade de vida, e que tiveram falha em tratamento conservador. ${ }^{2,14}$

Somente o artigo de Edwards et al. ${ }^{21}$ utilizou a subescala de catastrofização Coping Strategies Questionnaire (CSQ), que avalia a frequência de cognições e emoções catastróficas no contexto da dor. É um instrumento de medida de catastrofização comumente utilizado e possui boas propriedades psicométricas ( $a$ Cronbach=0,88). ${ }^{21}$

Os demais autores utilizaram a Escala de Catastrofização da Dor (PCS) elaborada por Sullivan et al. ${ }^{26} \mathrm{O}$ instrumento fornece um bom índice de catastrofização por meio das subescalas altamente correlacionadas de ruminação, magnificação e desamparo. A PCS tem forte consistência interna, apresentando $\alpha$ Cronbach: PCS-total, 0,87; PCS-ruminação, 0,87; PCSmagnificação, 0,66; e PCS-desamparo, 0,78. ${ }^{26}$

Tendo em vista que a PCS avalia 13 itens e possui intervalo de escores que varia de zero a 52, e que a subescala CSQ possui um intervalo de escores que varia somente de zero a seis, a comparação com os resultados do estudo de Edwards et al. ${ }^{21}$ deve ser realizada com cautela. A faixa restrita da subescala de catastrofização 
CSQ, juntamente com o número amostral reduzido, pode ter limitado a oportunidade de avaliar as mudanças na catastrofização durante o período de estudo.

Forsythe et al. ${ }^{22}$ usaram a PCS e não relataram mudança significativa no escore de catastrofização da dor ao longo de dois anos após a ATJ. Isso pode ter ocorrido devido à pequena população recrutada e com média da pontuação total da catastrofização de 9,8 48,7 no período pré-cirúrgico. Foi a menor pontuação no período que antecedia a ATJ encontrada nos artigos revisados. Dessa forma, os autores podem ter recrutado indivíduos que catastrofizavam pouco em relação os indivíduos dos outros estudos no período que antecedia a artroplastia. No entanto, essa informação não é precisa, visto que os autores não citam os critérios de inclusão e exclusão utilizados em seu estudo.

O sexo feminino foi maioria nos artigos revisados. Muitos estudos mostram que as mulheres relatam significativamente mais dor do que os homens. ${ }^{36-39}$ Keefe et al. ${ }^{38}$ relataram, em seu estudo, que as mulheres são mais propensas a catastrofizar a dor que os homens, e que há indicações de que as respostas de dor podem ser socializadas de forma diferente em homens e mulheres, favorecendo o desenvolvimento de um estilo catastrófico no sexo feminino desde a infância.

Nesse contexto dos fenômenos dolorosos, o modelo biopsicossocial defende que os fatores psicossociais têm importante papel na adequação dos indivíduos à dor, influenciando a funcionalidade. ${ }^{40}$ Assim, o indivíduo não é um mero receptor passivo de informação sensorial, mas a interpreta e reflete sobre ela, tendo contribuição ativa. ${ }^{41}$ Em suma, de acordo com esse modelo, a dor não é um fenômeno meramente sensorial, nem puramente psicológico, mas resultante da inter-relação entre fatores biológicos e psicossociais, estáveis e mutáveis que amplificam a informação sensorial e determinam o comportamento do indivíduo face à dor, afetando a noção de incapacidade dos mesmos. ${ }^{2}$ Tendo por base essa perspectiva teórica e com base nos resultados do presente estudo, já que a catastrofização da dor influencia diretamente o prognóstico e tratamento de idosos com OA de joelho, uma abordagem centrada nesse aspecto deve ser tomada. ${ }^{23,42,43} \mathrm{~A}$ escala de catastrofização da dor (PCS) pode ser facilmente administrada pelo terapeuta ao longo do exame físico pré-operatório. Nesse caso, indivíduos que apresentarem níveis elevados de catastrofização da dor em relação a dados normativos devem ser monitorados mais de perto. Dessa forma, intervenções adequadas nos períodos pré e pós-operatórios poderão ser implementadas, e o custo da cirurgia e da reabilitação podem ser diminuídos. ${ }^{22,24,34,43}$ A PCS, traduzida e adaptada para a população brasileira, obteve índices satisfatórios de consistência interna e de confiabilidade. Os autores recomendam sua utilização em ambientes clínicos pela sua fácil compreensão e boas propriedades psicométricas. ${ }^{44}$

O presente estudo possui uma limitação de heterogeneidade de amostra, visto que alguns dos artigos não analisaram somente a população idosa, mas também com idade entre 40 e 59 anos. Isso é bastante comum devido à dificuldade da coleta de dados, pois a população idosa apresenta particularidades clínicas e características próprias da idade. Dessa forma, alguns autores excluem essa população ou avaliam adultos jovens e idosos na mesma amostra, dificultando o entendimento de suas condições clínicas. ${ }^{45}$ No Brasil, há referências que apontam a faixa etária de 50 a 60 anos como sendo "quase-idosos", sendo importante estudálos para melhorar sua condição de vida após os 60 anos. ${ }^{45}$ Outra limitação se refere ao viés de idioma, já que foram incluídos apenas os artigos escritos em inglês e português, condição recorrente em revisões de literatura. ${ }^{41}$

\section{CONCLUSÃO}

Os resultados evidenciaram a importância da catastrofização da dor como preditora de 
recuperação após artroplastia total de joelho (ATJ). Segundo os estudos revisados, esse construto psicossocial se correlacionou positivamente com o tempo de permanência no hospital e com a intensidade de dor, e negativamente com funcionalidade após o procedimento cirúrgico. Assim, diante dos resultados obtidos pela

\section{REFERÊNCIAS}

1. Instituto Brasileiro de Geografia e Estatística. Comunicação Social em 01 de dezembro de 2009 [Internet]. [acesso em 10 Set. 2012]. Disponível em: http://www.ibge.gov.br/home/presidencia/noticias/ noticia_visualiza.php?id_noticia=1507\&id_pagina=1.

2. Haviv B, Bronak S, Thein R. The Complexity of Pain around the Knee in Patients with Osteoarthritis. Isr Med Assoc J 2013;15(4):244-247.

3. Wood A, Brock TM, Heil K, Holmes R. A Review on the Management of Hip and Knee Osteoarthritis. Int J Chronic Dis 2013:1-10.

4. Felson DT. An update on the pathogenesis and epidemiology of osteoarthritis. Radiol Clin North America 2004;42(1):1-9.

5. Hoogeboom TJ, den Broeder AA, de Bie RA, van den Ende CH. Longitudinal impact of joint pain comorbidity on quality of life and activity levels in knee osteoarthritis: data from the Osteoarthritis Initiative. Rheumatology (Oxford). 2013;52(3):543-6.

6. Hochberg, Haltman RD, April KT, Benkhalti M, Guyatt G, McGowan J, et al. American College of Rheumatology 2012. Recommendations for the Use of Nonpharmacologic and Pharmacologic Therapies in Osteoarthritis of the Hand, Hip, and Knee. Arthritis Care Res 2012;64(4):465-74.

7. Coimbra IB, Pastor H, Greve JMD, Puccinelli MLC, Fuller R, Cavalcanti FS, et al. Osteoartrite (Artrose): tratamento. Rev Bras Reumatol 2004;44(6):450-3.

8. Dunbar MJ. Subjective outcomes after knee arthroplasty [tese]. Acta Orthop Scand, Supl 2001;72(Supl 301):1-63.

9. Baker PN, van de Meulen JH, Lewsey J, Gregg PJ; National Joint Registry for England and Wales. The role of pain and function in determining patient satisfaction after total knee replacement. Data from the National Joint Registry for England and Wales. J Bone Jt Sur Ser B ,Br Vol 2007;89:893-900. revisão, recomenda-se que seja implementada a identificação precoce dos indivíduos que catastrofizam mais, o que pode implicar redução do tempo de recuperação e dos custos do pré e pós-procedimento cirúrgico, assim como melhora da qualidade de vida dos idosos que necessitam passar por essa intervenção.

10. Dieppe P, Murray D, Price A, Dodd C, Jenkins C, Pandit H. Persistent Knee pain after knee replacement for osteoarthritis. Osteoarthr Cartil 2010;18 Supl 2:S223.

11. Brander VA, Stulberg SD, Adams AD, Harden RN, Bruehl S, Stanos SP, Houle T. Predicting total knee replacement pain: a prospective observational study. Clin Orthop Relat Res 2003;416:27-36.

12. Hawker G, Wright J, Coyte P. Health-related quality of life after knee replacement. J Bone Jt Sur Ser A Am Vol 1998;80(2):163-73.

13. Ethgen $\mathrm{O}$, Bruyère $\mathrm{O}$, Richy $\mathrm{F}$, Dardennes $\mathrm{C}$, Reginster JY. Health-related quality of life in total hip and total knee arthroplasty. A qualitative and systematic review of the literature. J Bone Jt Sur Ser A Am Vol 2004;86(5):963-74.

14. Riddle DL, Keefe FJ, Na WT, McKee D, Attarian DE, Jensen MP. Pain coping skills training for patients with elevated pain catastrophizing who are scheduled for knee arthroplasty: a quasi-experimental study. Arch Phys Med Rehabil 2011;92:859-65.

15. Wesseling J, Welsing PN, Bierma-Zeinstra SM, Dekker J, Gorter KJ, Kloppenburg M, et al. Impact of self-reported comorbidity on physical and mental health status in early symptomatic osteoarthritis: the CHECK (Cohort Hip and Cohort Knee) study. Rheumatology (Oxford) 2013;52(1):180-8.

16. Keefe FJ, Caldwell DS, Queen K, Gil KM, Martinez S, Crisson JE, et al. Osteoarthritic knee pain: a behavioral analysis. Pain 1987;28(3):309-21.

17. Keefe FJ, Abernethy AP, Campbell LC. Psychological approaches to understanding and treating diseaserelated pain. Ann Rev Psychol 2005;56:601-30.

18. Venkataramanan V, Gignac MA, Dunbar M, Garbuz D, Gollish J, Gross A, et al. The importance of perceived helplessness and emotional health in understanding the relationship among pain, function, and satisfaction following revision knee replacement surgery. Osteoarthr Cartil 2013;21(7):911-7. 
19. Harden RN, Wallach G, Gagnon CM, Zereshki A, Mukai A, Saracoglu M, et al. The osteoarthritis knee model: psychophysical characteristics and putative outcomes. J Pain 2013;14(3):281-9.

20. Hawker GA, Gignac MA, Bladley E, Davis Am, French MR, Li Y, et al. A Longitudinal Study to Explain the Pain-Depression Link in Older Adults With Osteoarthritis. Arthritis Care Res 2011;63(10):1382-90.

21. Edwards RR, Havthornthwaite JA, Smith MT, Klick B, Katz JN, et al. Catastrophizing and depressive symptoms as prospective predictors of outcomes following total knee replacement. Pain Res Manag 2009;14(4):307-11.

22. Forsythe ME, Dunbar MJ, Hennigar AW, Sullivan MJ, Gross M, et al. Prospective relation between catastrophizing and residual pain following knee arthroplasty: Two-year follow-up. Pain Res Manag 2008;13(4):335-41.

23. Sullivan M, Tanzer M, Stanish W. Psychological determinants of problematic outcomes following total knee arthroplasty. Pain 2009;143(1-2):123-9.

24. Riddle DL, Wade JB, Jiranek WA, Kong X. Preoperative pain catastrophizing predicts pain outcome after knee arthroplasty. Clin Orthop Relat Res 2010;468(3):798-806

25. Fitzgerald JD, Orav EJ, Lee TH, Marcantonio ER, Poss R, Goldman L, et al. Patient quality of life during the 12 months following joint replacement surgery. Osteoarthr Cartil 2004;51(1):100-9.

26. Sullivan MJ, Bishop SR, Pivik J. The Pain Catastrophizing Scale: Development and validation. Psychol Assess 1995;7(4):524-32.

27. Jensen MP. A neuropsychological model of pain: research and clinical implications. J Pain 2010;11(1):2-12.

28. Campbell CM, Quartana PJ, Buenaver LF, Haythornthwaite JA, Edwards RR. Changes in situation-specific pain catastrophizing precede changes in pain report during capsaicin pain: a crosslagged panel analysis among healthy, pain-free participants. J Pain 2010;11(9):876-84.

29. Sullivan MJ, Thorn B, Haythornthwaite JA. Theoretical perspectives on the relation between catastrophizing and pain. Clin J Pain 2001;17(1):52-64.

30. Kovacs F, Noquera J, Abraira V, Royuela A, Cano A, Gil del Real MT, et al. The Influence of Psychological Factors on Low Back Pain-Related Disability in Community Dwelling Older Persons. Pain Med 2008;9(7):871-80.
31. Escobar A, Quintana JM, Bilbao A, Azkárate J, Güenaga JI, Arenaza JC, et al. Effect of patient characteristics on reported outcomes after total knee replacement. Rheumatology (Oxford) 2007;46(1):112-9.

32. Jones CA, Pohar S. Health-related quality of life after total joint arthroplasty: a scoping review. Clin Geriatr Med 2012;28(3):395-429.

33. Edwards RR, Cahalan C, Mensing G, Smith M, Haythornthwaite JA. Pain, catastrophizing, and depression in the rheumatic diseases. Nat Rev Rheumatol 2011;7:216-25.

34. Witvrouw E, Pattyn E, Almqvist KF, Crombez G, Accoe C, Camvier D, et al. Catastrophic thinking about pain as a predictor of length of hospital stay after total knee arthroplasty: a prospective study. Knee Surg Sports Traumatol Arthrosc 2009;17(10):1189-94.

35. Wade JB, Riddle DL, Thacker LR. Is pain catastrophizing a stable trait or dynamic state in patients scheduled for knee arthroplasty? Clin J Pain 2012; 28:122-8.

36. Davis MA. Sex differences in reporting osteoarthritic symptoms: a sociomedical approach. J Health Soc Behav 1981;22:298-310.

37. Felson DT. Epidemiology of osteoarthritis. In: Brandt KD, Doherty M, Lohmander LS, editors. Osteoarthritis. New York: Oxford University Press; 1998. p. 13-22.

38. Keefe FJ, Lefebre JC, Egert JR, Affleck G, Sullivan MJ, Caldwell DS. The relationship of gender to pain, pain behavior, and disability in osteoarthritis patients: the role of catastrophizing. Pain 2000;87(3):325-34.

39. Tonelli SM, Rakel BA, Cooper NA, Angstom WL, Sluka KA. Women with knee osteoarthritis have more pain and poorer function than men, but similar physical activity prior to total knee replacement. Biol Sex Differ 2011;2:1-12.

40. Enger-Yeger B, Dunn W. Relationship between pain catastrophizing level and sensory processing patterns in typical adults. Am J Occup Ther 2011;65:1-10.

41. Vissers MM, Bussmann JB, Verhaar JA, Busschbach JJ, Bierma-Zeinstra SM, Reijman M. Psychological factors affecting the outcome of total hip and knee arthroplasty: a systematic review. Osteoarthritis 2012;41(4):576-88.

42. Chapple CM, Nicholson H, Baxter D, Abbott HJ. Patient Characteristics That Predict Progression of Knee Osteoarthritis: a Systematic Review of Prognostic Studies. Arthritis Care Res 2011;63(8):1115-25. 
43. Sullivan M, Tanzer M, Reardon G, Amirault D, Dunbar M, Stanish W. The role of presurgical expectancies in predicting pain and function one year following total knee arthroplasty. Pain 2011;152(10):2287-93.

44. Lopes RA. Adaptação transcultural e análise das propriedades psicométricas da Escala de Catastrofização da Dor para a população idosa brasileira com dor lombar [dissertação]. Belo Horizonte: Universidade Federal de Minas Gerais, Escola de Educação Física; Fisioterapia e Terapia Ocupacional, Belo Horizonte, 2012.

45. Netto FLM. Aspectos biológicos e fisiológicos do envelhecimento humano e suas implicações na saúde do idoso. Pensar Prát 2004;7(1):75-84. 